\title{
Influencia de los procesos de reparación en las propiedades de los materiales de las carrocerías. Análisis de reparabilidad y selección del procedimiento adecuado
}

\author{
F. Mata*
}

\begin{abstract}
Resumen La práctica totalidad de los procesos de reparación de la chapa introducen tensiones residuales en el material, en tanto en cuanto recurren a técnicas de batido y deformación en frío o a calentamientos de forma local, y heterogeneidades como consecuencia de la aplicación de masillas de relleno, de propiedades mecánicas diferentes a las de la chapa original. La elección del proceso de reparación viene marcada por la severidad del daño y tiene implicaciones directas en el coste final de la reparación y, especialmente, en la fiabilidad del vehículo. Se analiza, en este trabajo, la variación del límite elástico en elementos de carrocería que han sufrido un impacto y posteriormente se han reparado recurriendo a diferentes procedimientos. Se presentan los resultados obtenidos para elementos de acero y dos procesos de reparación convencionales. El objetivo es determinar el proceso de reparación que afecta en menor medida a las propiedades iniciales del material. Se utiliza la técnica de simulación mediante elementos finitos como apoyo a los ensayos reales.
\end{abstract}

Palabras clave Carrocerías. Procesos de reparación. Resistencia a la tracción. Elementos finitos.

\section{Influence of the repair processes in the materials properties of the car bodies. Analysis of repair and selection of the suitable procedure}

Abstract

\begin{abstract}
The majority of the repair processes of the car bodies introduce residual tensions in the material, because the use of special work technologies, and heterogeneities as consequence of the application of putties, with different mechanical properties in comparison to the original sheet. The election of the repair process depends of the damage severity and it has important effects on the repair costs and the reliability of the vehicle. The variation of the elastic limit in elements of car body that have suffered an impact and later they have been repaired by different procedures is analyzed in this work. The results obtained for steel elements and two conventional processes of repair has been presented. The aim is to determine the repair process that produce a minor affect on the initial properties of the material. Finite elements simulation has been used to complete the tests.
\end{abstract}

Keywords Car bodies. Repair processes. Tensile strength. Finite elements.

\section{INTRODUCCIÓN}

Una de las aportaciones más importantes de la industria del automóvil ha sido el desarrollo de nuevos materiales para responder a las necesidades básicas de resistencia y ligereza ${ }^{[1]}$. De este modo, han aparecido paulatinamente materiales metálicos más ligeros y resistentes, materiales plásticos reforzados, etc.

En los procesos de fabricación de las carrocerías intervienen todo un conglomerado materiales de muy diversa naturaleza, como aceros aleados, plásticos, vidrios, etc., cada uno con un cometido y perfectamente integrados entre $s^{[2-3]}$. Los requerimientos de resistencia mecánica se consiguen con un conformado adecuado de la carrocería. Nos referimos a los pliegues, curvaturas y refuerzos, que contribuyen, desde la reducción de peso, al incremento de las propiedades mecánicas.

Los mayoría de los paneles exteriores de las carrocerías se obtienen por conformación de la chapa mediante procesos de laminación e

$\left(^{*}\right)$ Universidad de Castilla-La Mancha, Escuela Universitaria Politécnica de Almacén, Plaza Manuel Meca, 1, 13.400, Almadén (Ciudad Real), Tf: 926-264007, Fax: 926-264401, Francisco.MCabrera@uclm.es 
Influencia de los procesos de reparación en las propiedades de los materiales de las carrocerías. Análisis de reparabilidad y selección del procedimiento adecuado

F. MATA

hidroconformación. Este último procedimiento no introduce tensiones residuales en el material y consigue una uniformidad de propiedades muy deseable. Los elementos ya conformados se someten a diferentes tratamientos anticorrosivos y de acabado final.

Cuando un elemento de una carrocería recibe un golpe y se supera el límite elástico del material, aparece una deformación plástica (la chapa "se hunde") y, según la cuantía del esfuerzo, puede llegar incluso a la rotura del material. Es en el caso de deformación plástica cuando la zona afectada es susceptible de reparación, para devolverla a las cotas originales mediante diferentes procedimientos, siguiendo pautas de trabajo muy laboriosas. En caso de rotura, sólo hay dos posibilidades: o se sustituye completamente el elemento (por ejemplo una puerta, una aleta, etc.) o se efectúa una sustitución parcial, para lo cual es preciso recurrir a procesos de corte y soldadura.

Los profesionales chapistas ("artesanos de la conformación de materiales") se encargan de devolver la carrocería a las dimensiones y formas originales. Para ello, se valen de diferentes procesos de reparación (batido, conformado, desabollado neumático, sustitución parcial, estirado, enmasillado, etc.) entre los que puede optar en función de la gravedad del daño y del tipo de material a reparar ${ }^{[4]}$.

Un proceso completo de reparación de un panel exterior que ha sufrido un daño leve o medio (entendemos que el daño grave supone la sustitución parcial de la zona afectada o bien el cambio total del elemento) se compone de la siguiente secuencia de operaciones ${ }^{[1-4]}$ :

- lijado, hasta dejar la chapa desnuda en la zona afectada y alrededores,

- utilización de peines de formas, para evaluar el daño y facilitar la reconstrucción de la forma original,

- batido o desabollado, haciendo uso de herramientas y máquinas específicas (tases, martillos, tranchas, martillo de inercia, air-puller, etc.),

- igualación de superficies, con masilla de relleno,

- lijado y comprobación,

- limpieza y desengrasado,

- aplicación de aparejos e imprimaciones anticorrosivas,

- limpieza y desengrasado,

- aplicación de pinturas de acabado final,

- control de calidad (en todas las fases) ${ }^{[5]}$.

Cualquier proceso de reparación de una zona afectada siempre va a generar una discontinuidad (heterogeneidad) en las características originales del elemento de la carrocería, bien por aportación de otro material, por debilitamiento de la chapa, por tratamiento térmico local, etc. Lo ideal sería no tener que reparar.

Por otro lado, en el caso de daños fuertes en la estructura del vehículo, es muy importante que al finalizar la reparación la estructura "quede cuadrada", con las dimensiones o cotas originales y con la distribución de pesos original. Esta es una cuestión vital cuando se conduce a altas velocidades, ya que afecta a la estabilidad del vehículo y, en consecuencia, a su seguridad ${ }^{[6]}$.

Por tanto, en cualquier proceso de reparación o reconformado se modifican las propiedades iniciales de los materiales afectados. Se trata, pues, de evaluar la incidencia de estos procesos en las propiedades de los materiales, lo que sin duda puede aportar un criterio de decisión a la hora de reparar o sustituir por elementos nuevos. Además, permitirá tomar la decisión adecuada en cuanto al procedimiento a elegir para efectuar la reparación, dependiendo del tipo de daño y material, lo que tiene también gran interés desde el punto de vista económico.

\section{MATERIALES Y MÉTODOS}

El objetivo de este trabajo es analizar la incidencia de los diferentes procesos de reparación en las propiedades de los elementos de las carrocerías; con ello, se espera contribuir a seleccionar el proceso de reparación más adecuado en cada caso, tomando en consideración los criterios mecánicos.

En concreto, se estudian los procesos de desabollado o batido y desabollado con fase final de enmasillado para igualar las superficies. En esencia, se trata de dos procesos de trabajo de la chapa sin aportación de material y con aportación de un segundo material de características mecánicas diferentes. En el caso de enmasillado final, se aporta una masilla de relleno, generalmente de poliéster, de manera que la zona reparada queda marcada por la heterogeneidad, en tanto en cuanto está constituida por dos materiales diferentes que, incluso, pueden tener espesores variables, y la anisotropía, que tiene gran influencia en el comportamiento del conjunto, especialmente ante un nuevo choque o golpe.

Se ha tomado como referencia, en los dos casos, un panel exterior de carrocería fácilmente identificable y con una geometría estándar. Se trata de la aleta delantera de un turismo monovolumen, 
fabricada en chapa de acero AISI 1010 de 343 Mpa de límite de resistencia a la tracción y 0,8 mm de espesor.

Se prepararon un total de 7 probetas de dimensiones $0,12 \times 0,12 \mathrm{~m}^{2}$, de las que se obtuvieron probetas más pequeñas para los ensayos. Para ello, fueron necesarias 3 aletas nuevas, con el fin de que todas las probetas contasen siempre con la misma geometría (forma, nervaduras, etc.). Se ensayó una probeta de material nuevo, para contrastar el valor obtenido con el que ofrece el fabricante y al mismo tiempo refrendar la bondad del resto de ensayos. Para cada procedimiento de reparación se utilizaron 3 probetas, con el fin de determinar el valor medio. Con objeto de facilitar los trabajos de reparación, las probetas fueron cortadas mediante equipo de plasma una vez concluidas todas las operaciones sobre la pieza íntegra (aleta).

La deformación fue causada mediante gato hidráulico aplicando una carga constante de 120 $\mathrm{kg} / \mathrm{cm}^{2}$ durante $14 \mathrm{~s}$, consiguiendo de esta forma una deformación máxima de $9 \mathrm{~mm}$.

En los procesos de reparación se han utilizado las siguientes máquinas y herramientas:

- "proceso de desabollado o batido": tas, martillo de chapista, lima, trancha, palanca, peine de formas, lijadora rotoorbital neumática,....

- "proceso de enmasillado tras batido": juego de espátulas, lijadora rotoorbital, lijadora orbital, peine de formas.

Se ha utilizado masilla de poliester polifuncional con aplicación de $3 \%$ de catalizador y secado a temperatura ambiente durante $25 \mathrm{~min}$. Posteriormente, la superficie se lija mediante disco abrasivo de grano P 180.

Para realizar los ensayos de tracción se ha hecho uso de una máquina de ensayo (UNE 74-7492) ${ }^{[7]}$, que permite realizar tanto ensayos estáticos como dinámicos, con adquisición de datos monitorizada, según se aprecia en la Fig. 1.

Con el fin de modelar el proceso de deformación y la influencia del procedimiento de reparación se ha utilizado la herramienta de elementos finitos ANSYS. En la figura 2 se aprecia el tipo de elemento escogido y la densidad de mallado variable, en función de la curvatura de la aleta. Se han utilizado espesores de chapa de $0,6 \mathrm{~mm}, 1,0 \mathrm{~mm}$, $1,6 \mathrm{~mm}$ y $2,4 \mathrm{~mm}$ para el panel analizado. La tabla I presenta los valores de intensidad de carga empleados en la simulación en función del espesor de la chapa.

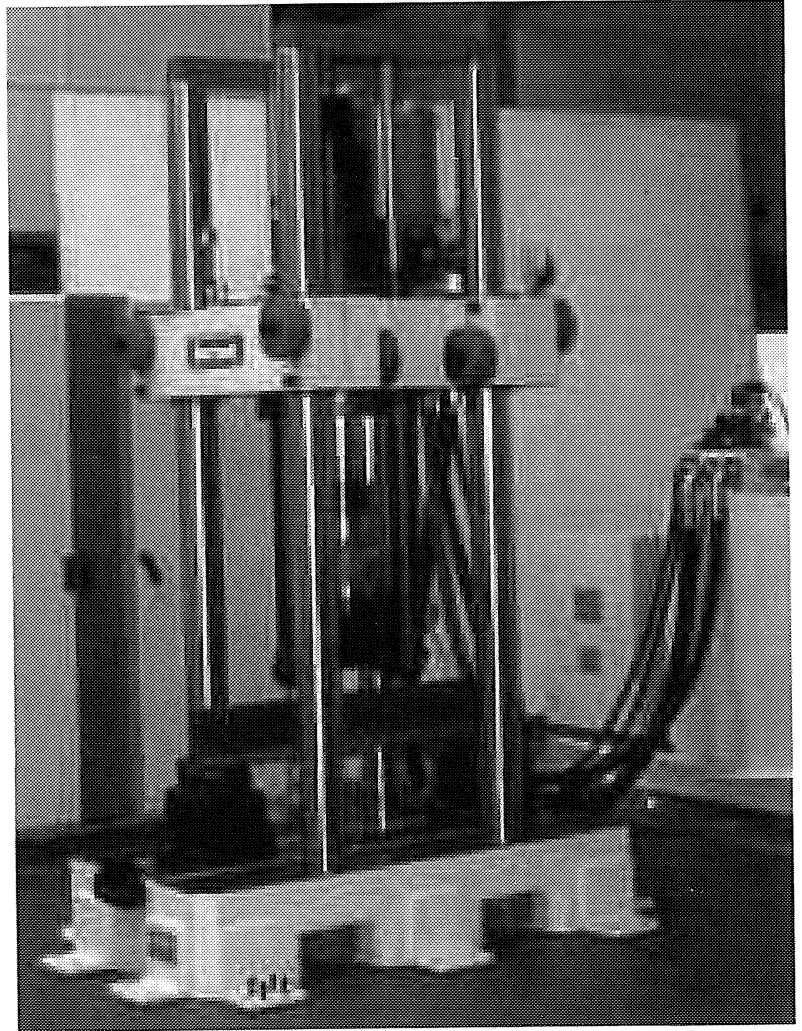

Figura 1. Máquina de tracción utilizada en los ensayos.

Figure 1. Machine used for tests.

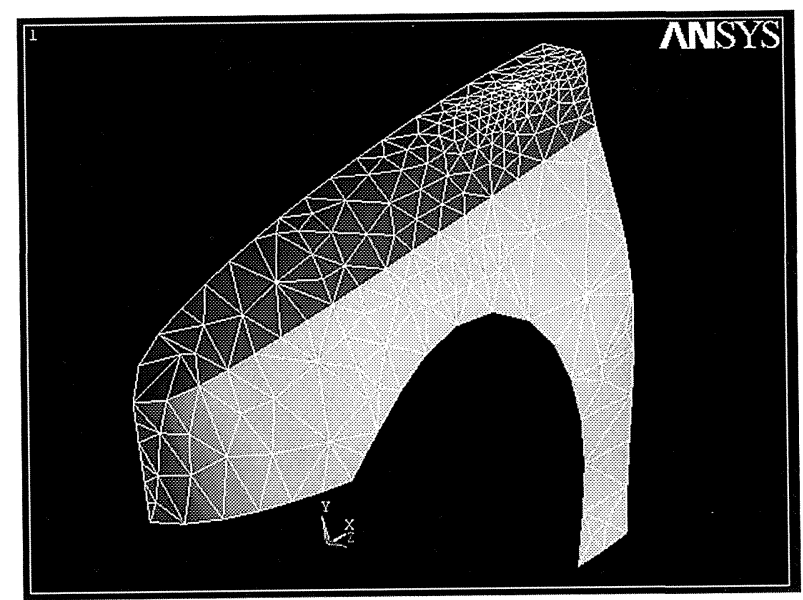

Figura 2. Modelo de la pieza construido en ANSYS.

Figure 2. Model constructed in ANSYS.

\section{RESULTADOS Y DISCUSION}

Tal y como se apuntó más arriba, el objetivo básico es determinar la influencia de los dos procesos de reparación estudiados en el valor del límite de resistencia a la tracción del acero.

La tabla II presenta los resultados de las medidas del límite elástico correspondientes al ensayo de tracción para las probetas reparadas por los dos 
Influencia de los procesos de reparación en las propiedades de los materiales de las carrocerías. Análisis de reparabilidad y selección del procedimiento adecuado

F. MATA

Tabla I. Intensidades de carga en función del espesor

Table I. Load intensities in function to the thickness

\begin{tabular}{lll}
\hline & INTENSIDAD(MPa) & \\
\hline & INT1 & 0,05 \\
ESPESOR 1 $(0,6 \mathrm{~mm})$ & INT2 & 0,1 \\
& INT3 & 0,4 \\
& INT4 & 0,8 \\
ESPESOR 2 $(1,0 \mathrm{~mm})$ & INT1 & 0,1 \\
& INT2 & 0,4 \\
\cline { 3 - 3 } ESPESOR 3 $(1,6 \mathrm{~mm})$ & INT3 & 0,8 \\
& INT4 & 1,6 \\
& INT1 & 0,4 \\
& INT2 & 0,8 \\
ESPESOR 4 $(2,4 \mathrm{~mm})$ & INT3 & 1,6 \\
& INT4 & 2,67 \\
& INT1 & 0,8 \\
& INT2 & 1,6 \\
& INT3 & 2,67 \\
& INT4 & 4 \\
\hline
\end{tabular}

Tabla II. Valores de límite elástico en probetas reparadas $(\mathrm{MPa})$

Table II. Values of elastic limit in repaired workpiece (MPa)

\begin{tabular}{|c|c|c|c|}
\hline Chapa & reparada ${ }^{(*)}$ & Chapa nueva ${ }^{(* *)}$ & Diferencia (\%) \\
\hline P 1 & 264 & 343 & 25 \\
\hline P 2 & 211 & 343 & 41 \\
\hline
\end{tabular}

${ }^{(*)}$ Media de tres medidas

${ }^{(* *)}$ Media del valor teórico y del valor medido

$P$ 1: desabollado o batido

P 2: enmasillado tras batido

procedimientos y la comparación, en términos de porcentaje, con el valor de una probeta nueva.

Como se puede observar, los dos procesos de reparación tienen una influencia negativa sobre el valor del límite elástico, reduciéndolo por encima de un $25 \%$ en el mejor de los casos. Precisamente, es el procedimiento de desabollado o batido simple el que resulta ser menos pernicioso, mientras que la aportación de masilla de poliéster en el segundo procedimiento origina una drástica reducción de la resistencia de las probetas, quedándose en aproximadamente el $60 \%$ de la resistencia inicial. Esto tiene una gran importancia desde el punto de vista práctico, especialmente si el vehículo sufre algún daño en las zonas reparadas con anterioridad, que tienen menor resistencia y que puede derivar, sin duda, en consecuencias negativas para la seguridad.
La figura 3 muestra de los valores obtenidos en los ensayos en las diferentes probetas para los dos procesos de reparación estudiados. La observación de las curvas de los ensayos permite evidenciar el comportamiento más frágil de la probeta que incorpora masilla de relleno, que rompe mucho antes que el acero y que reduce apreciablemente la resistencia del conjunto. La explicación se encuentra en que en el caso de utilizar masilla de relleno la chapa de acero está adelgazada (no se ha restituido el espesor inicial), de modo que la sección resistente equivalente es sensiblemente menor. Por otro lado, la contribución de la masilla a la resistencia a la tracción es prácticamente despreciable.

El conocimiento del límite elástico a tracción es fundamental, pues tiene una gran incidencia a la hora de restaurar la forma original de una chapa deformada. En estos casos, es preciso volver a deformarla, sobrecargándola por encima del límite elástico, en sentido contrario a la deformación original. Por tanto, influye directamente sobre el trabajo de deformación necesario para restituir las cotas originales.

En general, si los procesos de reparación conducen a resultados malos, es preciso sustituir en lugar de reparar. En nuestro caso, para la deformación inicial estimada, que corresponde con un daño severo-medio, sería más recomendable sustituir en lugar de reparar o, en todo caso, utilizar el primer procedimiento de reparación.

La utilización del modelo de elementos finitos en ANSYS nos ha permitido analizar el comportamiento del material para diferentes niveles de sobrecarga, obteniendo desplazamientos, tensiones residuales, etc., lo que sin duda puede ayudar a interpretar mejor el comportamiento dinámico de una carrocería al someterla a tests de choque. A título de ejemplo, la figura 4 representa la distribución de tensiones en un caso de precarga típico.

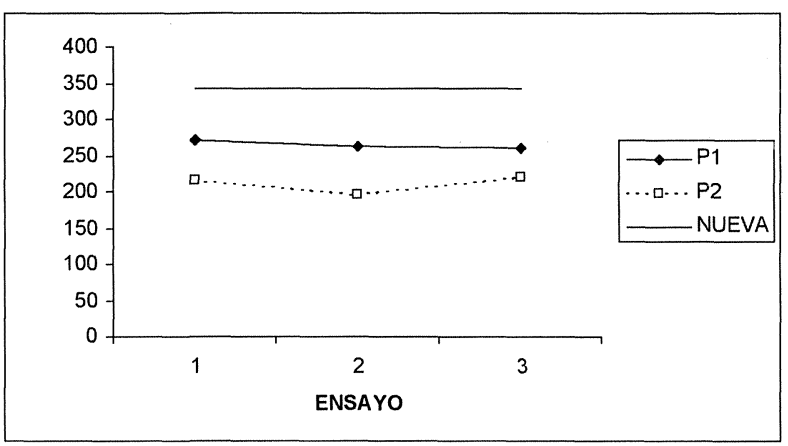

Figura 3. Comparativa del límite elástico.

Figure 3. Comparative of the elastic limit. 


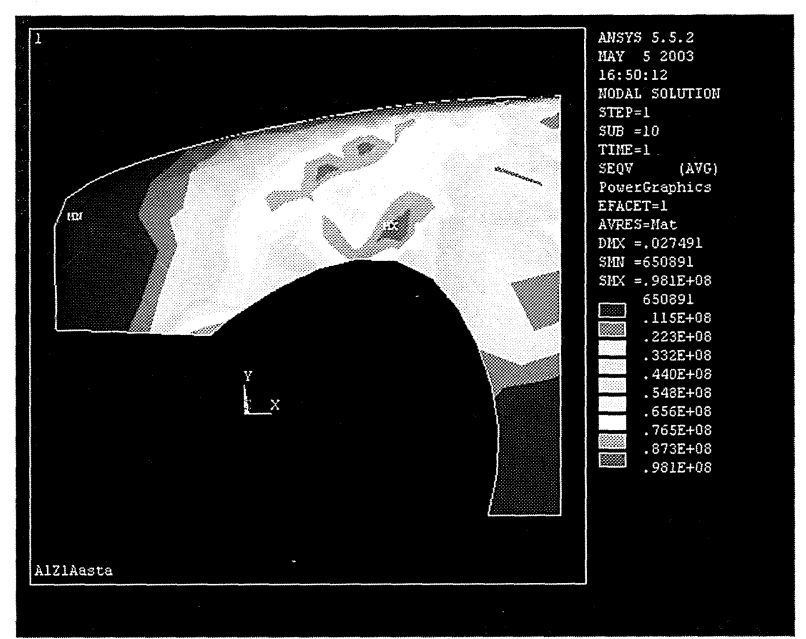

Figura 4. Tensiones de Von Mises.

Figure 4. Von Mises's tensions.

Se ha simulado el fenómeno de impacto sobre un panel exterior de carrocería (aleta). En el análisis se sustituye el cuerpo incidente por un conjunto de fuerzas y se parte de un estado de tensión-deformación nulo. El impacto se describe en dos pasos. En el primer paso, la carga aplicada sobre un área predefinida se hace variar desde un $0 \%$ de intensidad al $100 \%$ de la intensidad aplicada. En el segundo paso se establece la variación inversa (100\% al $0 \%$ ).

Las variables de entrada seleccionadas que definen el fenómeno físico del choque son el espesor del panel y la intensidad de la carga. Las variables de salida utilizadas son las necesarias para describir el estado de la chapa, es decir, los niveles de tensión-deformación y los niveles de energía, todas ellas medidas cuando la carga incide al $100 \%$ y cuando ésta ha cesado.

El objetivo de la simulación es establecer relaciones empíricas (basadas en la experiencia virtual de simulación numérica mediante elementos finitos) entre las variables de entrada (espesor, intensidad de carga) y las variables de salida (tensiones, deformaciones, energías). Estas relaciones servirán de base para establecer criterios de fallo con los que describir el comportamiento del modelo, en cada caso.

A continuación, como botón de muestra, se presentan algunas gráficas de seguimiento de errores para un ajuste de tipo logarítmico. La figura 5 muestra, comparativamente, los resultados de la experiencia virtual y los propuestos por el ajuste. El círculo representa el valor de energía residual de la chapa estimado en la simulación numérica y la cruz representa la estimación del ajuste propuesto.

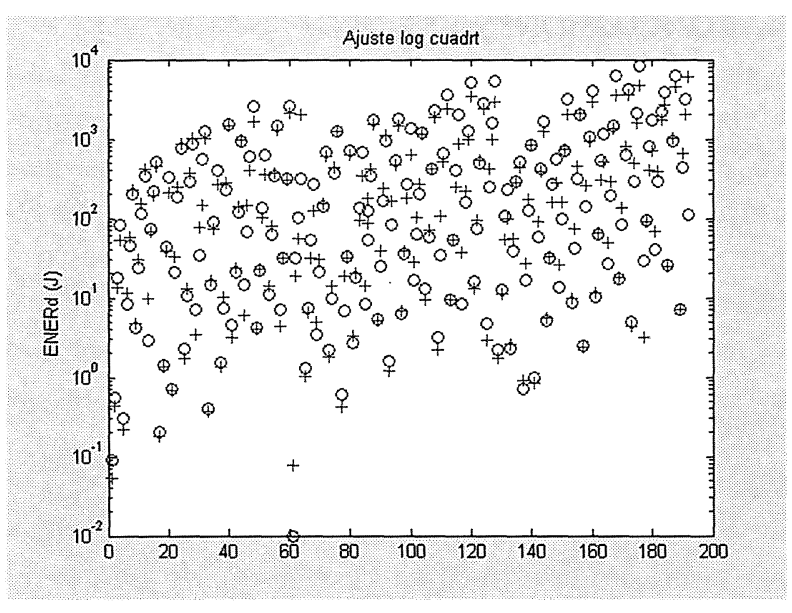

Figura 5. Representación de la energía residual de la chapa.

Figure 5. Representation of the residual energy.

En las Fig. 6 y 7 se muestran, respectivamente, los errores absolutos y relativos asociados al ajuste.

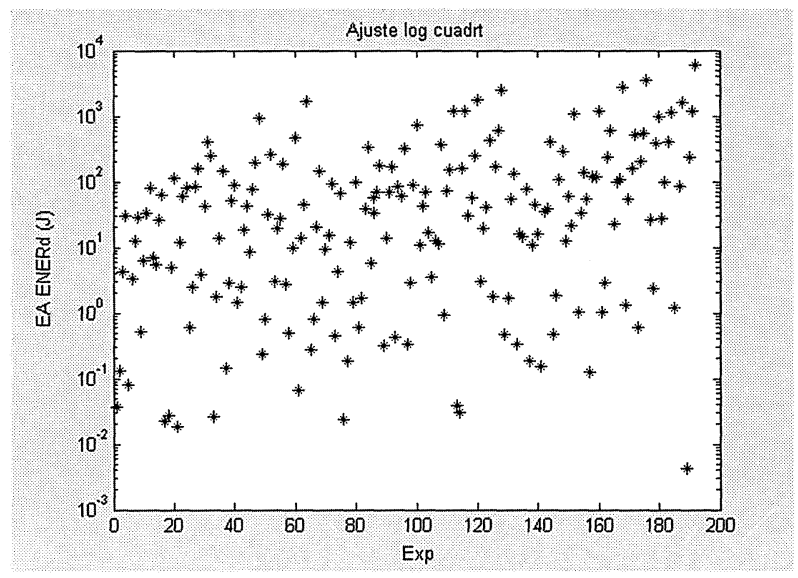

Figura 6. Representación del error absoluto.

Figure 6. Representation of the absolute error.

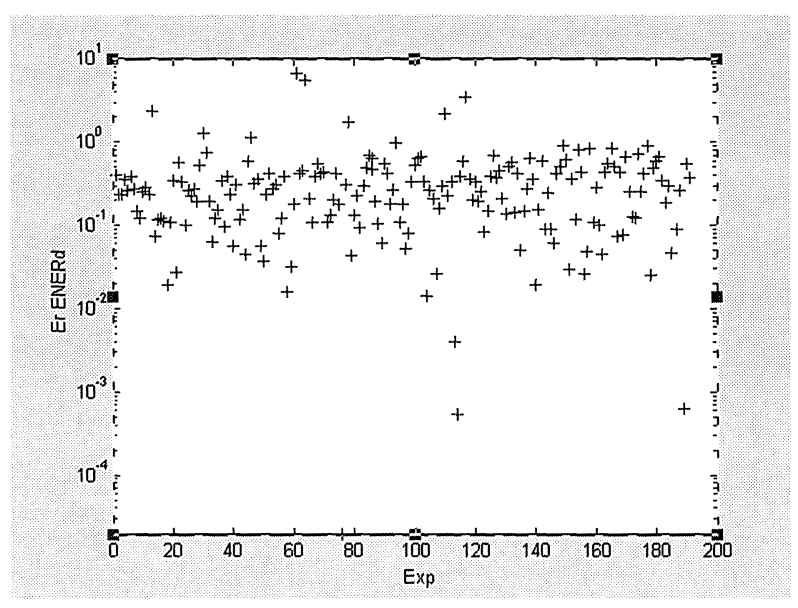

Figura 7. Representación del error relativo.

Figure 7. Representation of the relative error. 
Influencia de los procesos de reparación en las propiedades de los materiales de las carrocerías. Análisis de reparabilidad y selección del procedimiento adecuado

F. MATA

\section{CONCLUSIONES}

Cabe decir, finalmente, que este trabajo se enmarca en un proyecto de investigación mucho más amplio en el que se analizan otros procesos de reparación (aplicación de calor, soldadura, estiramiento en bancada, etc.), otros materiales (aceros especiales, aluminio, materiales compuestos) y el estudio de otras propiedades específicas como la resistencia al doblado o al choque, para poder tener una idea más amplia del problema.

En todo caso, ha quedado de manifiesto el interés múltiple de esta línea de trabajo: de una parte interés técnico, que revierte en la seguridad del automóvil y, de otra, interés económico, al ofrecer al profesional de la reparación criterios científicos para discernir el proceso más adecuado (aquel que garantice la máxima calidad en la reparación en un tiempo óptimo).

Por otra parte, en virtud de los resultados de este trabajo, entendemos que el profesional de la reparación debe utilizar con mesura los productos de relleno de naturaleza no metálica, reduciendo su uso exclusivamente para igualar superficies y mejorar el acabado una vez se haya trabajado (batido, estirado, recogido) convenientemente la chapa. En ningún caso, la masilla sustituirá al desabollado o batido de los paneles metálicos.
Finalmente, la simulación mediante elementos finitos permite obtener de forma rápida y poco costosa un referente de resultados que son contrastados con los ensayos reales. El cálculo de las deformaciones permitirá estimar el tipo de daño y marcará criterios de reparabilidad a los profesionales del sector.

\section{REFERENCIAS}

[1] F. MATA, E. SÁNCHEZ, Monografías didácticas del curso Tecnologías del automóvil, Servicio de Publicaciones de la Escuela Universitaria Politécnica de Almadén, España, 2001, pp. 162-175.

[2] R. GARROTE, Reparación de vehículos: chapistería, (Autor Editor), Barcelona, España, 1994, pp. 42-74.

[3] D. Carle, G. Blount, Rev. Mater. 20 (1999) 267-272.

[4] E. Águeda, J.L. García, Elementos metálicos y sintéticos, Ed. Paraninfo, Madrid, España, 1998, pp. 23-59.

[5] G. Subner, G. Greiner, S. Augustiniack, Rev. ComputerAided 36 (2004) 425-436.

[6] M. Cascajosa, Ingeniería de vehículos, Ed. Tebar, Madrid, España, 2004, pp. 221-230.

[7] J.P. Davim, Ensaios mecânicos e tecnológicos, Estante Ed., Aveiro, Portugal, 1992, pp. 87-95. 